\title{
Standpunkt
}

Wie Unternehmen die Aufgaben von Umweltverbänden übernehmen

\section{Unheilvolle Allianz oder letzte Chance?}

In Zukunft werden Unternehmen bisher wirtschaftsfremde

Naturbilder verwenden, um neue Märkte zu erschließen. Weil sie

damit entscheidend das Denken und Handeln der Konsumenten

prägen, werden die Wirtschaft und ihre Eliten über die Zukunft

der Natur und des Naturschutzes mitbestimmen.

Von Nicole Lüdi

D er Natur- und Umweltschutzbewegung gelang es, die Vorstellung, die wir von Natur und Umwelt haben, zu prägen. Diese Bilder waren ursprünglich dazu gedacht, die Menschen für die Natur und deren Schutz zu mobilisieren. Zunehmend geraten sie aber in den Fokus der Wirtschaft, die ihr enormes Potenzial erkannt hat.

\section{Naturbilder werden durch die Wirtschaft erschlossen}

Sah die Wirtschaft bis jetzt Natur als zu nutzende Ressource, wird sie sich in Zukunft immer stärker bei den Bildern der Natur- und Umweltbewegungen bedienen. Die Geschäfte mit der Sehnsucht nach Natur (Tourismus, Wellness, Gesundheit), der Hoffnung, die in Nachhaltigkeit gesetzt wird (Design, Technologie, Architektur), und den Ängsten vor Naturveränderungen (Schutz, Versicherungen) stehen erst am Anfang.

So führt der Erfolg des Natur- und Umweltschutzes in der Vermittlung ihrer Naturbilder paradoxerweise dazu, dass Natur in den kommenden Jahren vollends zur konsumistischen Spielwiese wird. Um die Inszenierung der Natur werden sich in Zukunft die Marketingabteilungen kümmern. Die ehemals verfemte Wirtschaft wird, den Sehnsüchten und Ängsten entsprechend, die passenden Bilder liefern - und so den Alltag mit Naturbildern fluten.

Damit übernimmt die Wirtschaft zwei zentrale Funktionen von Naturschutzor- ganisationen. Erstens schafft sie Aufmerksamkeit für Naturthemen. Zweitens schützt sie Natur, indem sie beispielsweise Ressourcen schonende Produkte entwickelt oder Landschaften für den Tourismus bewahrt.

\section{Wohlstand segmentiert die Beziehung zur Natur}

Wenn allerdings der Markt den Naturund Umweltschutz übernimmt, werden Einkommen und Vermögen über unsere Beziehung zur Natur bestimmen. Denn dann muss die Beziehung zur Natur gekauft werden.

Die Reichen können die vermittelten Naturbilder real werden lassen - sei es, indem sie sich in echte Natur begeben können, in Städten leben, die dank der Rückkehr der Natur hohe Lebensqualität bieten, oder sich Produkte kaufen, die allen ethischen Ansprüchen genügen. Außerdem können sie sich optimal vor Naturrisiken absichern. Menschen der unteren Schichten hingegen werden sich nur in eingeschränkter Form eine Beziehung zur Natur leisten können.

In den letzten Jahren bestand die Beziehung zwischen Wirtschaft und Naturschutz vor allem in Blamingstrategien seitens der Nichtregierungsorganisationen (NRO). Sie deckten Missstände bei Unternehmen auf, machten diese publik und erreichten damit, dass sich die Konsumenten entrüstet von der jeweiligen Firma abwendeten - was diese zwang, ihr Verhalten zu ändern.
In Zukunft werden aber immer mehr NROs die wirtschaftliche Elite für ihre Zwecke einspannen. Dies wird möglich sein, weil die Wirtschaftsführer, allein schon aus Marketing-Gründen, ein großes Interesse daran haben, Gutes zu tun. Aber das ist nicht der einzige Grund: Immer mehr Manager verspüren eine Sehnsucht nach Sinnerfüllung. Das werden die NROs zu nutzen wissen.

\section{Unheilvolle Allianz von Umweltverbänden und Wirtschaft?}

Neu gestaltete Angebotssituationen würden die Konsumenten quasi zwingen, nachhaltige Produkte zu kaufen. Mit diesem Strategiewechsel wird ein neues Kapitel im Naturschutz aufgeschlagen. Versuchte man früher, über die Konsumenten das Verhalten der Unternehmen $\mathrm{zu}$ ändern, wird in Zukunft über die Unternehmen der Kunde zum guten Konsumenten gemacht. Damit bezwecken die NROs eine Effizienzsteigerung bei der Erreichung ihrer Natur- und Umweltschutzziele. Die Wirtschaftselite wird also auch auf Einladung der NROs in Zukunft immer wichtiger beim Natur- und Umweltschutz.

Durch das Näherrücken der NROs zur Wirtschaft wird der Schutz der Natur zu einer Eliteangelegenheit. Jene NROs, die diese Strategie wählen, verlassen die Grass-Roots-Bewegungen. Ihre ursprüngliche Legitimation als Stellvertreter der Zivilgesellschaft und ihre Glaubwürdigkeit als unabhängige Akteure geraten unter Druck und erodieren. Diese NROs sehen sich künftig in zunehmendem Maße selbst durch Blamingstrategien von $\mathrm{Ni}$ schenorganisationen der Natur- und Umweltbewegung herausgefordert.

I AUTORIN + KONTAKT

Nicole Lüdi ist Forscherin am GDI Gottlieb Duttweiler Institute.

GDI Gottlieb Duttweiler Institute, Langhaldenstraße 21, Postfach 531, 8803 Rüschlikon/Zürich, Schweiz. Tel.: +41 447246111 E-Mail: nicole.luedi@gdi.ch. 
(c) 20I0 Authors; licensee IÖW and oekom verlag. This is an article distributed under the terms of the Creative Commons Attribution Non-Commercial No Derivates License (http://creativecommons.org/licenses/by-nc-nd/3.o/), which permits unrestricted use, distribution, and reproduction in any medium, provided the original work is properly cited. 